\title{
Method for the Detailed Characterization of Cosputtered Inorganic Luminescent Material Libraries
}

\author{
Evert P. J. Merkx*(1) and Erik van der Kolk \\ Luminescence Materials, Delft University of Technology, Mekelweg 15, 2629 JB Delft, The Netherlands
}

Supporting Information

ABSTRACT: Understanding the behavior of combinatorially developed luminescent materials requires detailed characterization methods that have been lacking thus far. We developed a device for directly surveying the luminescent properties of thin-film libraries created through combinatorial gradient sputter deposition. Step-scan recorded excitation-, emission- and luminescence decay spectra of a thin-film library were resolved and combined with EDX measurements on the same film, relating composition to luminescent properties. This technique was applied to a single-

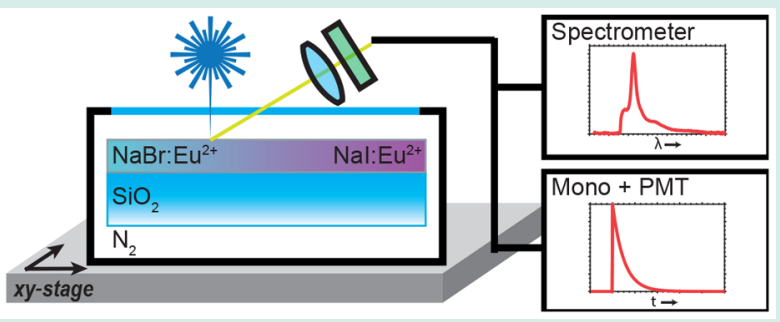
substrate gradient thin-film library of $\mathrm{NaBr}_{0.73} \mathrm{I}_{0.27}$ to $\mathrm{NaBr}_{0.09} \mathrm{I}_{0.91}$, doped with $6.5 \%$ to $16.5 \% \mathrm{Eu}^{2+}$. This gradient film closely followed Vegard's law, with emission fluently shifting from 428 to $439 \mathrm{~nm}$. In comparison, pure $\mathrm{NaBr}: \mathrm{Eu}^{2+}$ showed emission at $428 \mathrm{~nm}$ and $\mathrm{NaI}: \mathrm{Eu}^{2+}$ at $441 \mathrm{~nm}$. Luminescence decay measurements demonstrated a great degree of concentration quenching in the gradient film. From these measurements we could conclude that an optimized phosphor would most efficiently luminesce when close to $\mathrm{NaI}: \mathrm{Eu}^{2+}$. This gradient film confirmed that the method presented in this work allows to both study and optimize luminescent behavior in a broad range of host- and dopant systems.

KEYWORDS: inorganic phosphors, combinatorial screening, sputter deposition, luminescence

\section{INTRODUCTION}

Combinatorial synthesis finds broad application in many fields, but in the case of phosphor synthesis, only a limited number of reports exist. This lack of past works can be explained by that combinatorial methods, when applied to conventional searches like the search for white light LEDs, usually converge to known phosphors. ${ }^{1}$ In addition, the analysis methods used for combinatorially synthesized libraries of luminescent materials are often limited to (color-filtered) charge-coupled device (CCD) photography of a library under UV illumination, ${ }^{2-10}$ substrate-scanning with color filters to isolate emission peaks, ${ }^{11,12}$ or cathode luminescence with no information on low energy excitations. ${ }^{13}$ These coarse characterizations still have to be followed by powder synthesis to fully analyze the luminescent properties of a material of interest.

In this Research Article, we present a novel method that enables the detailed characterization of a luminescent composition library, based on combinatorial gradient deposition of thin-films using DC/RF magnetron sputtering. This gradient is realized by taking advantage of the low mobility of sputtered adatoms when depositing without substrate heating. ${ }^{13}$ The presented method is capable of resolving positiondependent structural and compositional information, as well as photoluminescent excitation, emission, and decay spectra. This combination allows us to link the position-dependent structural and compositional information to the recorded luminescence properties, thereby retrieving direct composition-property relations.
Determination of the structure and composition is done with step-scan X-ray diffraction (XRD) for crystallinity and phase determination. Energy-dispersive X-ray spectroscopy (EDX) is used to resolve the local composition of the gradient thin-film. Photoluminescence measurements are done using a combination of laser excitation and an $x y$-stepping stage, with luminescence captured by either a CCD spectrometer, or a photomultiplier tube (PMT) coupled to a digitizer.

In binary halides, substitution of anions can lead to a fluent shifting of emission wavelength between the two mixed compounds, in line with Vegard's law. For instance, $\mathrm{KCl}_{1-x} \mathrm{Br}_{x}$ crystals doped with $\mathrm{Eu}^{2+}$ have shown this fluent shifting of emission. ${ }^{14}$ Therefore, to illustrate the capabilities of the method, we have chosen a continuously varying mixed-phase library of $\mathrm{NaBr}: \mathrm{Eu}^{2+}$ and $\mathrm{NaI}: \mathrm{Eu}^{2+}$ as an example. While the individual phosphors have previously been researched, ${ }^{14}$ the behavior of the mixed-phase phosphor has not yet been studied. The end points of this library are therefore wellknown, while the mixed-phase in between provides new data on the behavior of mixed binary halides. The combination of luminescent, compositional and structural information is employed to see if these two phosphors will also display behavior following Vegard's law. Furthermore, the possibility of optimizing luminescent behavior in terms of quantum efficiency is explored with this library.

Received: May 4, 2018

Revised: August 17, 2018

Published: October 2, 2018 


\section{RESULTS AND DISCUSSION}

Library Creation and Method for Characterization. To provide an illustration of the combinatorial synthesis and characterization route, $\mathrm{NaBr}, \mathrm{NaI}$ and $\mathrm{Eu}$ were magnetron cosputtered at a $90^{\circ}$ angle from each other on a $5 \times 5 \mathrm{~cm}^{2} \mathrm{UV}$ fused silica substrate. Figure $1 \mathrm{a}$ and $1 \mathrm{~b}$ shows the layout of the
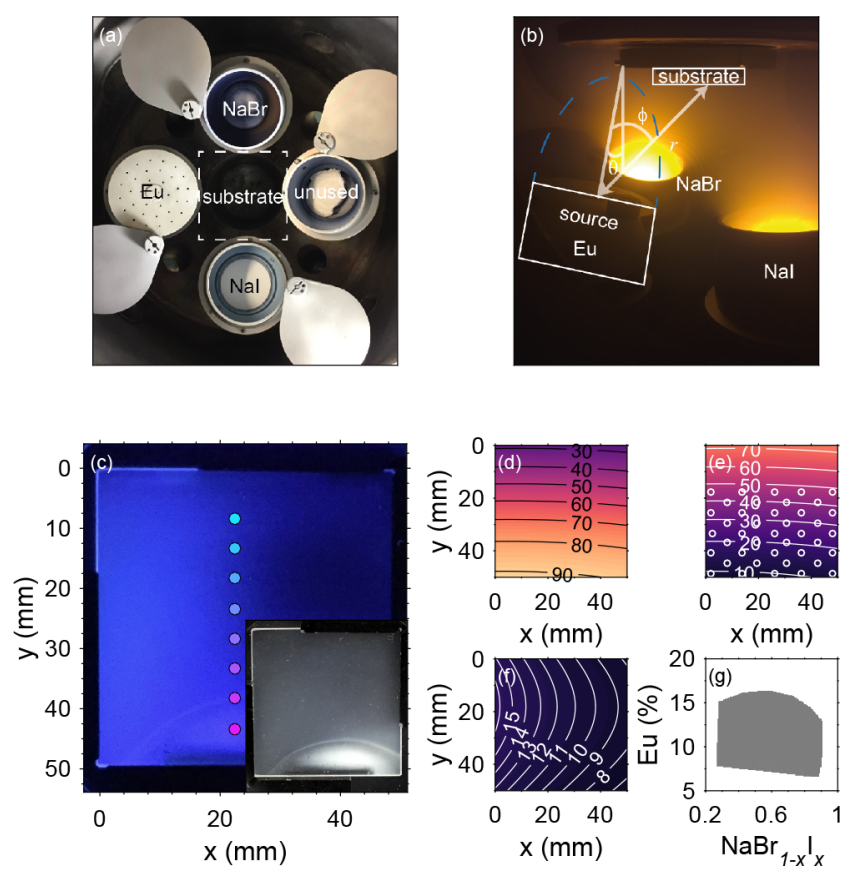

Figure 1. Compositional library of a gradient of $\mathrm{NaBr}$ to $\mathrm{NaI}$ doped with varying concentrations of Eu. (a) Photograph of the layout of the sputtering sources used to fabricate the gradient library. The source with $\mathrm{Eu}$ has a mask applied to reduce the sputtering yield, as sputtering at a lower power leads to an unstable plasma. This and all subsequent images have been oriented with $\mathrm{NaI}$ always depicted at the bottom, $\mathrm{NaBr}$ at the top and Eu to the left. (b) Photograph of the sputtering chamber during deposition. Overlaid is a schematic of the parameters used in eq 1 . The dashed lines are an illustration of the elongated spherical distribution of material sputtered from the Eu source. (c) Photograph of the sample under UV excitation, and (inset) white light illumination. Colored dots show where XRD measurements were taken. ( $\mathrm{d}, \mathrm{e}, \mathrm{f}) \mathrm{EDX}$ data across the substrate displaying atomic concentrations of $\mathrm{I}$ and $\mathrm{Br}$ and doping concentration of Eu, respectively. The white circles in panel e mark the locations where EDX measurements were taken. (g) Area showing the span of $\mathrm{NaBr}-\mathrm{NaI}-\mathrm{Eu}$ compositions covered by the substrate.

sputtering chamber prior to deposition and during deposition, respectively. UV fused silica was chosen since it does not show luminescence, contrary to other glass types. ${ }^{15}$ As with $\mathrm{Tm},{ }^{16}$ $\mathrm{Eu}$ sputtered together with binary alkali halides readily forms its divalent state, without the need for any additional oxidizing gases or heat treatments. The library was therefore deposited at room temperature using an inert $\mathrm{Ar}$ atmosphere, without further treatment. In addition, being $\mathrm{SiO}_{2}$ of high purity, it will not have overlap with the EDX lines for $\mathrm{Na}, \mathrm{Br}$, I and $\mathrm{Eu}$. Because of the hygroscopic nature of $\mathrm{NaBr}$ and $\mathrm{NaI}$, the asdeposited film was transported from the sputtering chamber to a nitrogen-filled glovebox using an airtight container under vacuum. In the glovebox, the sample (Figure 1c) was transferred to a $\mathrm{N}_{2}$-filled, airtight sample holder with a UV fused silica or polyimide foil window for optical or XRD measurements, respectively.
Figures $1 \mathrm{~d}$ to $1 \mathrm{f}$ show the elemental concentrations based on EDX measurements at 40 locations and an interpolation between these points.

Direct EDX measurements of the Eu concentration carry a high uncertainty. Contrary to $\mathrm{Eu}$, the ratio of $\mathrm{Na}$ to $\mathrm{Br}$ and I can be repeatedly measured with good certainty across the film. The composition of the film should be charge-neutral. The Eu concentration can therefore be derived from the measured ratio of $\mathrm{Na}$ to $\mathrm{Br}$ and $\mathrm{I}$ by setting the ratio of $\mathrm{Eu}^{2+}$ and $\mathrm{Na}^{+}$to $\mathrm{Br}^{-}$and $\mathrm{I}^{-}$equal to unity.

The interpolation is obtained by fitting with the surfacesource evaporation equation. The surface-source evaporation equation for a single source is based on the mass deposited on the substrate per unit area $\mathrm{d} M_{\mathrm{S}} / \mathrm{d} A_{\mathrm{S}}$, given by ${ }^{13,17}$

$$
\frac{\mathrm{d} M_{\mathrm{S}}(x, y)}{\mathrm{d} A_{\mathrm{S}}}=M_{\mathrm{T}} \frac{(n+1) \cos ^{n} \phi(x, y) \cos \theta}{2 \pi r^{2}(x, y)}
$$

Here, $M_{T}$ is the total mass exiting the sputtering source, $n$ determines the sharpness of the elongated sphere of material being sputtered from the source, $\phi$ is the angle between the source normal and a line extending from the center of the source to a position on the substrate, $\theta$ is the angle between the source normal and the substrate normal, and $r$ is the distance between the source and any position on the substrate's surface. These parameters are illustrated in Figure $1 b$. Fitting the concentration $c_{j}$ of a material $j \in i$ when using $N$ target materials $i$ can be accomplished with

$$
c_{j}(x, y)=\frac{\left(\frac{\mathrm{d} M_{\mathrm{s}}}{\mathrm{d} A_{\mathrm{s}}}\right)_{j}}{\sum_{i}\left(\frac{\mathrm{d} M_{\mathrm{s}}}{\mathrm{d} A_{\mathrm{s}}}\right)_{i}}
$$

where the fitting parameters are the respective $n_{i}$ and $M_{\mathrm{T}, i}$ of the sputtering sources.

The resultant $c_{j}$ for $j=\mathrm{I}, \mathrm{Br}$, and $\mathrm{Eu}$ are shown in Figure $1 \mathrm{~d}-1 \mathrm{f}$, respectively.

The sputtering yield of $\mathrm{NaI}$ is larger than that of $\mathrm{NaBr}$. The iodide concentration therefore follows the distribution yielded by the gun with $\mathrm{NaI}$, which leads to the semicircles seen in Figure 1d. Figure 1g brings all EDX results together and shows that this single substrate comprises a variation of $6.5 \%$ to $16.5 \% \mathrm{Eu}$, and a variation of $\mathrm{NaBr}_{0.73} \mathrm{I}_{0.27}$ to $\mathrm{NaBr}_{0.09} \mathrm{I}_{0.91}$. Across the substrate, this means an average slope of $0.05 \%$ / $\mathrm{mm}$ from $\mathrm{NaBr}$ to $\mathrm{NaI}$ and an average slope in Eu-doping percentage of $0.15 \% / \mathrm{mm}$ (all concentrations mentioned are in atomic percent).

A custom computer-controlled dual $x y$-translation stage that is synchronized with an optical parametric oscillator (OPO) laser and a data-acquisition system, referred to as the XYscanner, was developed for mapping the luminescent properties across the thin-film surface. A schematic of the entire characterization system is shown in Figure 2. Light ranging from 193 to $2600 \mathrm{~nm}$ with $0.3 \mathrm{~nm}$ line width generated by a $100 \mathrm{~Hz}$ (3-5 ns fwhm pulses) OPO laser is guided toward the stage to serve as an excitation source. The laser light is passed through a diaphragm to compensate for fluctuations in the position of the laser spot on the thin-film when stepping across wavelengths. A UV fused silica plate placed at a $45^{\circ}$ angle reflects $4 \%$ of this incident light toward a powermeter that is used to correct for wavelength- and time-dependent laser power fluctuations. Following excitation, luminescence passes through a long-pass filter to remove reflected laser light and is 


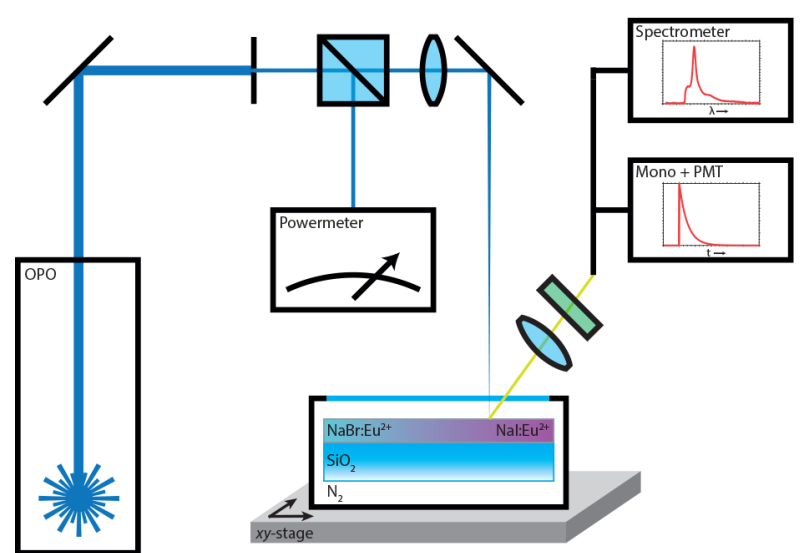

Figure 2. Schematic of the XY-scanner setup consisting of an OPO laser, a powermeter, an $x y$-translation stage containing the substrate and a fiber-optic CCD spectrometer or a monochromator with PMT. A laser spot diameter of about $70 \mu \mathrm{m}$ is used to not damage the sample with the highest available laser power of $1.8 \mathrm{~W}$.

focused into an optical fiber that guides the light to a detector. This detector can either be a CCD spectrometer for emissionand excitation measurements, or a monochromator coupled to a PMT (Mono+PMT) connected to a digitizer for decay measurements. Excitation- and emission measurements produced with the XY-scanner show excellent agreement with what is measured with conventional methods, as shown in Figure S1.

Luminescent Alkali-Halide Library. Figure $3 \mathrm{a}$ and $3 \mathrm{~b}$ show the emission $\left(4 \mathrm{f}^{6} 5 \mathrm{~d}\left[\mathrm{t}_{2 \mathrm{~g}}\right] \rightarrow 4 \mathrm{f}^{7}\right)$ of the $\mathrm{NaI}-\mathrm{NaBr}: \mathrm{Eu}^{2+}$ thin-film library under laser excitation at $350 \mathrm{~nm}$. Scanning over the film with the laser yields emission spectra per position on the film. This data can be summarized in an RGB color plot, shown in the inset of Figure $3 \mathrm{a}$, which closely resembles the photograph shown in Figure 1c. This color-coded image is obtained by converting the measured emission spectra to CIEcoordinates and translating those to RGB values.

Figure 3 a shows that the emissions have a fluent transition from $428 \mathrm{~nm}$ at the $\mathrm{NaBr}$-rich side, to $438 \mathrm{~nm}$ at the NaI-rich side of the film. A closer look at the emission reveals that it is actually comprised of two bands and a broad (fwhm $>0.8 \mathrm{eV}$ ) sideband of low intensity, possibly comprised of multiple smaller bands. In the further discussion, this broad $(>0.8 \mathrm{eV})$ low energy emission is not shown due to its low intensity, and since it is most likely comprised of many higher-order defects. Figure $3 \mathrm{~b}$ illustrates this presence of multiple bands. We can see a second band at higher wavelengths developing when moving toward increased Eu concentration, while the other band stays centered at the same position. This qualitative analysis can be made more quantitative by deconvoluting the emission into two separate Gaussians. The central wavelengths of these Gaussians are displayed in Figure $3 c$ and $3 d$, with relative areas in Figure $3 \mathrm{e}$ and $3 \mathrm{f}$ and bandwidths in Figure $3 \mathrm{~g}$ and $3 \mathrm{~h}$. The emission of the narrow $(0.16-0.18 \mathrm{eV})$ and intense band at 428-438 nm (Figure 3c, 3e, and 3g) closely resembles the semicircles caused by the higher sputtering yield of $\mathrm{NaI}$ over $\mathrm{NaBr}$, as seen in Figure 1d. The broader (0.34$0.38 \mathrm{eV}$ ) emission's central wavelength (Figure $3 \mathrm{~d}, 3 \mathrm{f}$, and $3 \mathrm{~h}$ ) follows the increasing Eu concentration, moving toward lower energy and increasing in prominence with higher Eu concentration.

The luminescence quantum yield $\eta_{\mathrm{QY}}$ is an important measure for the performance of a phosphor, as it indicates the presence of luminescence quenching, for instance due to an overly high dopant concentration. The observed intensity (Figures $3 \mathrm{e}$ and $3 \mathrm{f}$ ) is not a direct measure of $\eta_{\mathrm{QY}}$, because the intensity also depends on the absorption, which in turn depends on the thickness of the film. A measure for $\eta_{\mathrm{QY}}$ independent of the luminescence intensity is the decay time.

Figure 4a shows individual luminescent decay spectra across the substrate. These spectra were measured with a slit width such that all light from 415 to $445 \mathrm{~nm}$ is collected. The exact locations of these measurements are displayed in Figure $4 \mathrm{~b}$. Because of the nonexponential nature of the measured decays, a mean decay time ${ }^{18}$

$$
\tau_{\text {mean }}=\frac{\int_{0}^{\infty} t I(t) \mathrm{d} t}{\int_{0}^{\infty} I(t) \mathrm{d} t}
$$
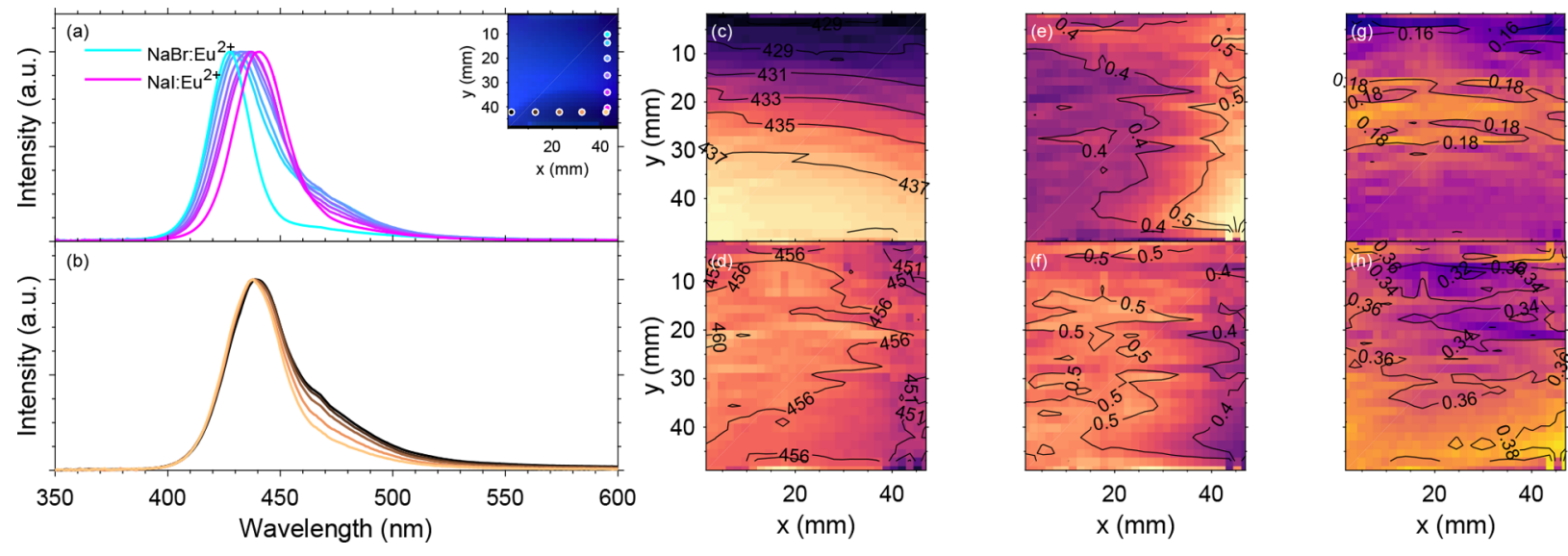

Figure 3. Relation between position and luminescence properties on the thin-film. (a) Normalized emission spectra $\left(\lambda_{\mathrm{ex}}=350 \mathrm{~nm}\right.$, taken at the locations marked by the blue-to-pink points shown in the inset) across the $\mathrm{NaBr}-\mathrm{NaI}$ gradient with comparable Eu concentration. Pure $\mathrm{NaBr}$ : $\mathrm{eu}^{2+}$ and $\mathrm{NaI}: \mathrm{Eu}^{2+}$ are also shown. (b) Normalized emission spectra across the $\mathrm{Eu}^{2+}$ gradient with identical $\mathrm{Br} / \mathrm{I}$ ratio $\left[\lambda_{\mathrm{ex}}=350 \mathrm{~nm}\right.$, taken at the locations marked by copper-colored points running right-to-left shown in the inset of panel a]. Respective (c, d) central wavelengths (in nm), (e, $f$ ) normalized areas (w.r.t. the total area of the emission), and (g, h) bandwidths (fwhm, in eV) of the two deconvoluted Gaussians (adjusted- $R^{2}>$ 0.9993). 

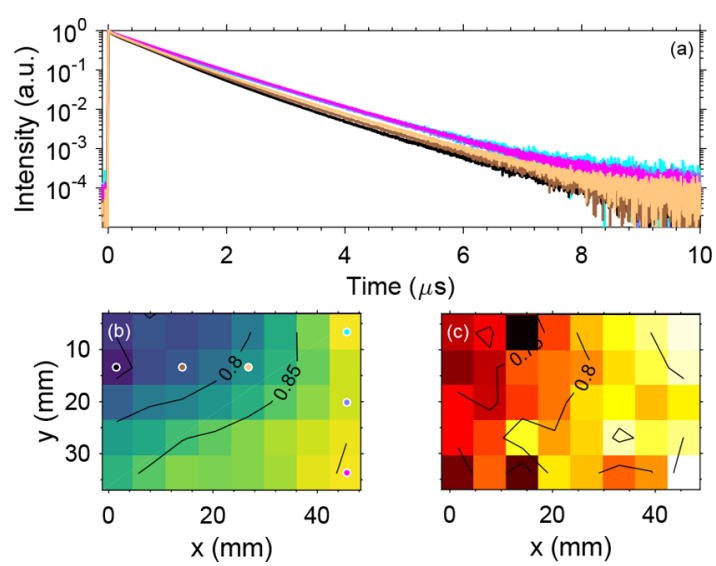

Figure 4. Decay characteristics of the $\mathrm{NaI}-\mathrm{NaBr}: \mathrm{Eu}^{2+}$ measured at $430 \pm 15 \mathrm{~nm}$. (a) Individual decay spectra, the blue-to-pink series run from $\mathrm{NaI}$ to $\mathrm{NaBr}$ rich, with a low $\mathrm{Eu}$ concentration, the coppercolored lines run from high to low $\mathrm{Eu}$ concentration, with approximately equal $\mathrm{NaBr}$ and $\mathrm{NaI}$ content. The exact locations where the spectra were taken are shown in (b), where also a full overview of the mean decay times (in $\mu \mathrm{s}$ ) is provided. (c) Estimated quantum yields based on a tail-fitting as explained in the main text (adjusted- $R^{2}>0.98$ ).

is determined. Here, $I(t)$ is the emission intensity at time $t$ after a laser pulse. $\tau_{\text {mean }}$ makes comparison between decay spectra possible, even if the exact behavior of the decay is unknown. Figure $4 \mathrm{~b}$ shows a mapping of $\tau_{\text {mean }}$.

The quantum yield of each composition can be estimated using a fitting of the tail of the decay spectrum. Fitting a single exponential to the tail of the decay spectra yields the decay time for an isolated ion $\tau_{\text {tail }}{ }^{18}$ Alkali-halides with a low europium doping are known to have near unity quantum yield at room temperature. ${ }^{19}$ It is therefore possible to relate the mean decay time to an estimated quantum yield as

$$
\eta_{\mathrm{QY}}=\frac{\tau_{\text {mean }}}{\tau_{\text {tail }}}
$$

This estimated quantum yield is shown in Figure 4c. Comparison between Figures $4 b$ and $4 c$ shows that the mean decay time does not directly correlate with quantum efficiency. The determined values should therefore not be compared per position, but per composition, as will be discussed in the following.

Using the XY-scanning technique, important luminescence properties of the film at room temperature are determined, while EDX measurements provide the composition of the same film. Since these measurements have the position of the film in common, these measurements can be combined to directly relate the deconvoluted emission, decay times and estimated quantum yields to the composition. These compositionproperty relationships, which leave out irrelevant position information, are shown in Figure 5.

Figure 5a shows that the high-energy band shifts in concert with the $\mathrm{Br}$ to I ratio, from $428 \mathrm{~nm}$ at $\mathrm{NaBr}_{0.73} \mathrm{I}_{0.27}$ to $438 \mathrm{~nm}$ at $\mathrm{NaBr}_{0.09} \mathrm{I}_{0.91}$. From Figure 3a, we know that thin-films of pure $\mathrm{NaBr}: \mathrm{Eu}^{2+}$ and $\mathrm{NaI}: \mathrm{Eu}^{2+}$ show a dominant emission at 428 and $441 \mathrm{~nm}$, respectively. These emissions are attributed to substitutional defects, where $\mathrm{Eu}^{2+}$ replaces $\mathrm{Na}^{+}$combined with a charge-compensating defect outside of the first coordinating sphere. ${ }^{14}$ Between $\mathrm{NaBr}$ and $\mathrm{NaI}$, the only changes in this first coordinating sphere are the type of the
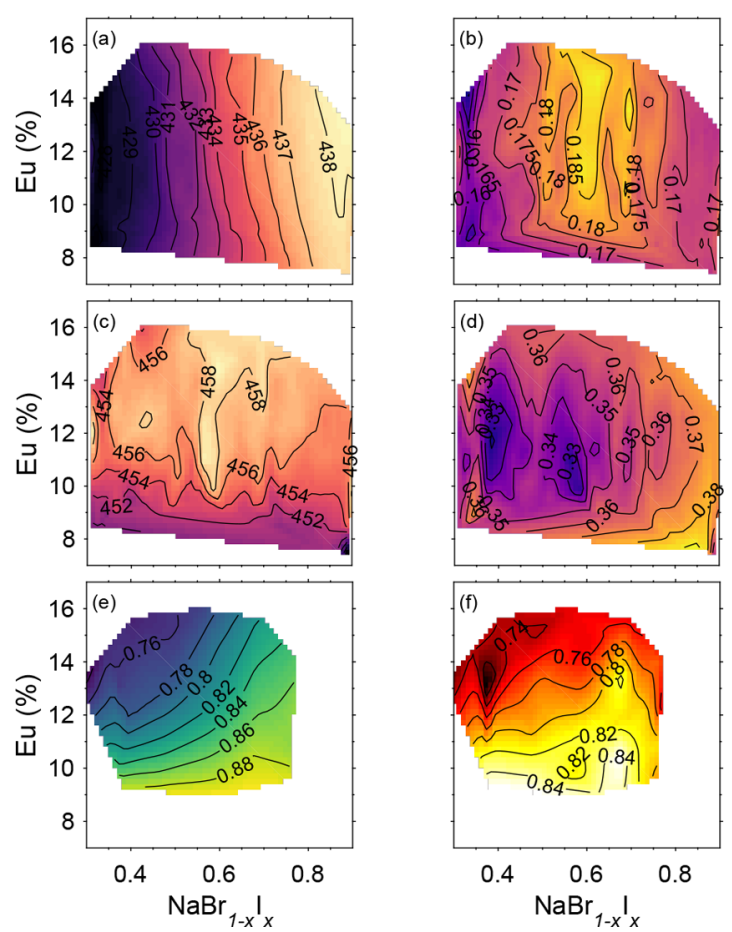

Figure 5. Relation between material composition and luminescence properties. (a) The emission wavelengths (in $\mathrm{nm}$ ) and (b) bandwidths (in $\mathrm{eV}$ ) as presented in Figure $3 \mathrm{c}$ and $3 \mathrm{~g}$ directly related to the composition as presented in Figure 1d to $1 \mathrm{f}$. (c) The emission wavelengths (in $\mathrm{nm}$ ) and (d) bandwidths (in $\mathrm{eV}$ ) as presented in Figure $3 \mathrm{~d}$ and $3 \mathrm{~h}$ related to the composition. (e) The decay time (in $\mu \mathrm{s})$ and (f) estimated $\eta_{\mathrm{QY}}$ from Figure $4 \mathrm{~b}$ and $4 \mathrm{c}$ related to the composition data from Figure $1 \mathrm{~d}$ to $1 \mathrm{f}$.

anions (becoming more electronegative from $\mathrm{Br}$ to $\mathrm{I}$ ) and the distance of the anions (having a larger ionic radius from $\mathrm{Br}$ to I). From a well-mixed phase that follows Vegard's law we would therefore expect the emission to change fluently from $\mathrm{NaBr}$ at $428 \mathrm{~nm}$ to $\mathrm{NaI}$ at $441 \mathrm{~nm}$, regardless of $\mathrm{Eu}$ concentration.

Another consequence of this mixed phase is that Europium becomes statistically coordinated with the surrounding anions. Because of this variation in the direct surroundings of $\mathrm{Eu}$, the emission peak broadens. This broadening is shown in Figure $5 b$. The emission of the substitutional defect in pure $\mathrm{NaBr}: \mathrm{Eu}^{2+}$ has a bandwidth of $0.13 \mathrm{eV}$, where in $\mathrm{NaI}: \mathrm{Eu}^{2+}$ it has a bandwidth of $0.16 \mathrm{eV}$. This difference leads to the maximal bandwidth in Figure $5 \mathrm{~b}$ not being displayed at $\mathrm{NaBr}_{0.5} \mathrm{I}_{0.5}$, but shifted slightly closer to $\mathrm{NaI}$, at $\mathrm{NaBr}_{0.43} \mathrm{I}_{0.57}$.

The emission band at lower energy (Figure 5c) might be attributed to a more complex defect. Previous research attributed a defect emitting at $453 \mathrm{~nm}$ in $\mathrm{NaBr}: \mathrm{Eu}^{2+}$ to a $\mathrm{EuBr}_{2}$-type precipitate, and emission at $461 \mathrm{~nm}$ in $\mathrm{NaI}: \mathrm{Eu}^{2+}$ to a EuI 2 -type precipitate. ${ }^{14}$ The emission shown in Figure $5 c$, supported by the larger bandwidth shown in Figure 5d, might therefore also be attributed to a $\mathrm{EuBr}_{2}-$ or $\mathrm{EuI}_{2}$-type precipitate, or a mixture thereof. The band's emission center shifts to lower energies with increasing Eu concentration. This redshifting might be a consequence of reabsorption of higher energy emission, which becomes more profound with more absorbing centers present.

Figure 5e shows that for the substitutional defect a decrease in Eu concentration leads to an increase in decay time. Because of the strong correlation with $\mathrm{Eu}$ concentration, we can 
conclude that a high degree of concentration quenching is present in our sample. For comparison, the highest $\tau_{\text {mean }}$ of $0.89 \mu \mathrm{s}$ is observed for $\mathrm{NaBr}_{0.36} \mathrm{I}_{0.64}: 9 \% \mathrm{Eu}$, where pure $\mathrm{NaBr}: \mathrm{Eu}^{2+}$ with a low Eu-doping would show $\tau_{\text {tail }}=1.05 \pm$ $0.1 \mu \mathrm{s}$ and $\mathrm{NaI}: \mathrm{Eu}^{2+}$ would show $\tau_{\text {tail }}=1.05 \pm 0.03 \mu \mathrm{s}$, as can be found in the Supporting Information. Lowering the concentration of Eu would increase the decay time, and thus the luminescence quantum yield. This relation is confirmed by Figure 5f. In Figure $5 f$ we can also see that the estimated quantum yield for compounds closer to $\mathrm{NaI}: \mathrm{Eu}^{2+}$ rises more quickly with $\mathrm{Eu}^{2+}$ concentration than for those closer to $\mathrm{NaBr}: \mathrm{Eu}^{2+}$. We can therefore expect that $\mathrm{NaI}: \mathrm{Eu}^{2+}$-type compounds will yield a brighter phosphor, when fully optimized for photoluminescent yield.

From Figure 6, we see that the fluent shifting behavior is not limited to the emission bands, but also occurs in the excitation

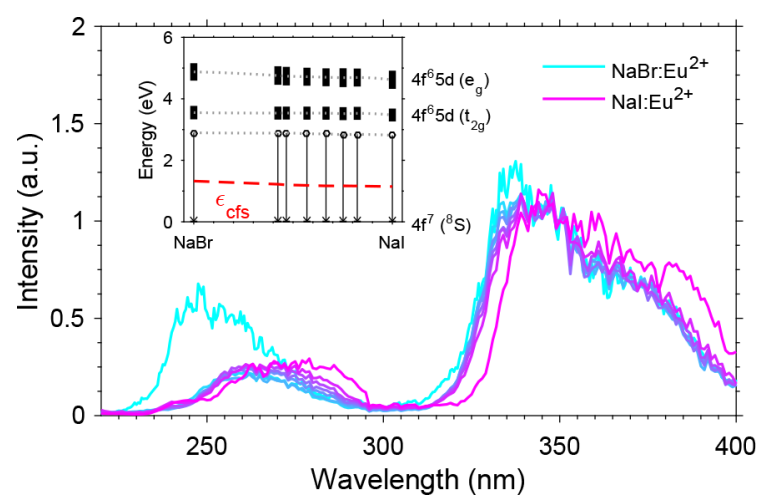

Figure 6. Normalized (at $\lambda_{\mathrm{ex}}=350 \mathrm{~nm}$ ) excitation spectra of the substitutional defect, taken at $\lambda_{\mathrm{em}}=420 \mathrm{~nm}$ to minimize influence of other defects. Spectra were recorded at the same positions as shown in Figure 3a. Excitation spectra of pure $\mathrm{NaBr}: \mathrm{Eu}^{2+}$ and $\mathrm{NaI}: \mathrm{Eu}^{2+}$ are shown in purple and light blue. The inset schematically shows the energy of the $5 \mathrm{~d}$ energy levels. Arrows show the observed emission maximum. The centers of the $e_{g}$ and $t_{2 g}$ levels are taken as the center of gravity (first moment) and their bandwidths as the second moment of their respective excitation bands. ${ }^{14}$ Dotted lines are drawn through the centers of the $e_{g}$ and $t_{2 g}$ levels and the emission energies as a guide to the eye. The crystal field splitting is shown as a red dashed curve.

bands $\left(4 \mathrm{f}^{7}\left[{ }^{8} \mathrm{~S}_{7 / 2}\right] \rightarrow 4 \mathrm{f}^{6} 5 \mathrm{~d}^{1}\right)$. The crystal field splitting $\epsilon_{\mathrm{cfs}}$ decreases when moving from $\mathrm{NaBr}$ to $\mathrm{NaI}$. This decrease is a result of the larger distance between $\mathrm{Eu}^{2+}$ and the surrounding halogen ions due to the larger ionic radius of I ions compared to $\mathrm{Br}$ ions. ${ }^{20}$ However, the emission for $\mathrm{NaI}: \mathrm{Eu}^{2+}$ is still at lower energy than for $\mathrm{NaBr}: \mathrm{Eu}^{2+}$. Hence, we can conclude that the shifting of the emission band from the substitutional defect is mainly dominated by a drop in the centroid energy, because the d-orbitals of the $\mathrm{Eu}^{2+}$ ion can expand further when surrounded with $\mathrm{I}^{-}$than when surrounded with $\mathrm{Br}^{-}$.

As final confirmation of the existence of a well-mixed phase, XRD measurements should show diffraction peaks fluently shifting from $\mathrm{NaBr}$, with lattice parameter $a=2.987 \AA$, to NaI, with $a=3.235 \AA$. This fluent shift can be seen in Figure 7 .

Figure 7 also shows a broadening of the diffraction peaks when moving from $\mathrm{NaBr}_{0.14} \mathrm{I}_{0.86}$ to $\mathrm{NaBr}_{0.52} \mathrm{I}_{0.48}$. This broadening has a similar origin as the broadening observed Figure $5 \mathrm{~b}$. As with the broadening of the emission, the broadening of the diffraction peaks is a consequence of the lattice spacing becoming more variable when more equal amounts of $\mathrm{I}$ and $\mathrm{Br}$ are included.

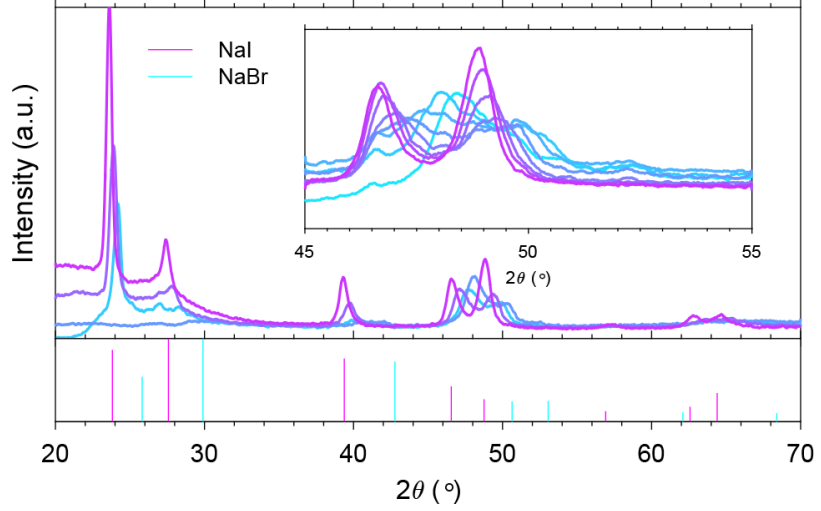

Figure 7. Diffractograms taken across the substrate following the colors as shown in Figure $1 \mathrm{c}$, which correspond to $\mathrm{NaBr}_{0.52} \mathrm{I}_{0.48}$ (blue) to $\mathrm{NaBr}_{0.14} \mathrm{I}_{0.86}$ (purple). The purple pattern underneath is reference pattern no. 1820477 for $\mathrm{NaI}$ and the blue lines are pattern no. 1400153 for $\mathrm{NaBr}$. The inset shows a close-up from $45-55^{\circ} 2 \theta$ of the shifting of the diffraction peaks from $\mathrm{NaI}$ to $\mathrm{NaBr}$. Relative peak intensities of the reference and measured diffractograms differ, since our measurements were done with constant-area X-ray illumination, where the reference is done with constant intensity.

\section{CONCLUSION}

We have shown that it is possible to create a luminescent thinfilm composition- and concentration material library. In a single gradient film the Eu concentration changes from $6.5 \%$ to $16.5 \%$ and the host composition from $\mathrm{NaBr}_{0.73} \mathrm{I}_{0.27}$ to $\mathrm{NaBr}_{0.09} \mathrm{I}_{0.91}$. Using the XY-scanning characterization method it is now possible to directly relate composition to photoluminescent emission-, excitation-, and decay properties. The method can generally be applied to a wide variety of material libraries with composition-, concentration-, and thickness gradients, not limited to a single doping or host precursor target.

The luminescence of $\mathrm{NaI}: \mathrm{Eu}^{2+}$ and $\mathrm{NaBr}: \mathrm{Eu}^{2+}$ has previously been explored, but the behavior of these phosphors in a mixed state was still unknown. Therefore, a $\mathrm{NaI}-\mathrm{NaBr}-\mathrm{Eu}^{2+}$ gradient thin-film served as an example for this method. The excitation and emission observed for the end points of the gradient, $\mathrm{NaI}: \mathrm{Eu}^{2+}$ and $\mathrm{NaBr}: \mathrm{Eu}^{2+}$, agree with what has previously been observed. ${ }^{14}$ In the mixed compound, we have seen that the $\mathrm{NaBr}-\mathrm{NaI}$ gradient closely follows Vegard's law, which is expressed as a fluent shift in luminescence from $428 \mathrm{~nm}$ when $\mathrm{NaBr}$-rich, to $438 \mathrm{~nm}$ when NaI-rich. This following of Vegard's law is further confirmed by XRD measurements, which show a fluent shifting of the diffraction peaks. These shifts agree with what is expected when moving between the two pure-phase compounds, as also observed in $\mathrm{KCl}_{1-x} \mathrm{Br}_{x}: \mathrm{Eu}^{2+}$ crystals, where $x$ was varied from 0 to $1 .^{14}$

From decay measurements, we can conclude that the deposited film exhibited concentration quenching but that, when optimized for photoluminescent yield, compounds closer to $\mathrm{NaI}: \mathrm{Eu}^{2+}$ will yield a brighter luminescent material than compounds close to $\mathrm{NaBr}: \mathrm{Eu}^{2+}$.

In conclusion, the XY-scanning method presented in this work makes it possible to systematically analyze a broad range of host compositions and dopant concentrations to study the behavior of luminescent materials, using only a single deposition. The results from the thin-film phosphors are highly comparable to those observed with traditional powderbased techniques. Hence, the method may provide a toolbox 
capable of accelerating the discovery and optimization of phosphors for a wide range of applications such as lighting, display and scintillation, but also for solar-conversion, afterglow, and many other types of photoactivated materials.

\section{EXPERIMENTAL PROCEDURES}

The $\mathrm{NaBr}-\mathrm{NaI}: \mathrm{Eu}^{2+}$ gradient thin-film was fabricated in an AJA Orion 5 magnetron sputtering system with a base pressure of $1 \times 10^{-9}$ bar. A $5 \mathrm{~cm}$ diameter Eu metal target $(99.99 \%$, Demaco) and $5 \mathrm{~cm}$ diameter $\mathrm{NaBr}$ and $\mathrm{NaI}$ pressed-powder targets (both $99.99 \%$, Alfa Aesar, pressed at $36 \mathrm{MPa}$ for $5 \mathrm{~min}$ ) were simultaneously sputtered at a $90^{\circ}$ angle from each other at room temperature in a $4 \times 10^{-2} \mathrm{mbar}$ Ar atmosphere $(6 \mathrm{~N}$ purity, $32 \mathrm{sccm}$ flow rate) onto a $5 \times 5 \mathrm{~cm}^{2}$ UV fused silica substrate (PGO). Prior to deposition, the substrate was cleaned for $15 \mathrm{~min}$ in an ultrasonic bath with a solution of soapy water, after which it was rinsed with acetone, ethanol and DI-water, and left to dry in an oven at $200{ }^{\circ} \mathrm{C}$ for more than $1 \mathrm{~h}$. The substrate and substrate carrier were loaded into the sputtering chamber while both were $>100{ }^{\circ} \mathrm{C}$ to prevent moisture contamination. The $\mathrm{NaBr}(\mathrm{RF}), \mathrm{NaI}(\mathrm{RF})$, and $\mathrm{Eu}$ (DC) sputter powers were fixed at $25(0.04 \mathrm{~nm} / \mathrm{s}), 31(0.08$ $\mathrm{nm} / \mathrm{s})$, and $10 \mathrm{~W}\left(<1 \times 10^{-3} \mathrm{~nm} / \mathrm{s}\right)$ respectively. The $\mathrm{Eu}$ target's chimney was masked using a stainless steel mask, with a pattern of concentric holes $1.2 \mathrm{~mm}$ in diameter, blocking $99 \%$ of the surface of the chimney (shown in Figure 1a). This finally led to a film with an estimated thickness of $0.86 \mu \mathrm{m}$ in the center, $0.9 \mu \mathrm{m}$ at the side of the film closest to $\mathrm{NaI}$ and 0.8 $\mu \mathrm{m}$ at the side of the film closest to $\mathrm{NaBr}$. Deposition happened at room temperature, without any further heat treatment. Using the same process, two reference films with only (1) $\mathrm{NaBr}: \mathrm{Eu}^{2+}$ or (2) $\mathrm{NaI}: \mathrm{Eu}^{2+}$ were fabricated: (1) sputter settings fixed at $40 \mathrm{~W}$ for $\mathrm{NaBr}(\mathrm{RF}, 0.08 \mathrm{~nm} / \mathrm{s}), 10 \mathrm{~W}$ for $\mathrm{Eu}\left(\mathrm{DC},<1 \times 10^{-3} \mathrm{~nm} / \mathrm{s}\right)$ and $(2) \mathrm{NaI}$ at $35 \mathrm{~W}$ (RF, 0.13 $\mathrm{nm} / \mathrm{s})$ and $\mathrm{Eu}$ at $10 \mathrm{~W}\left(\mathrm{DC},<1 \times 10^{-3} \mathrm{~nm} / \mathrm{s}\right)$. All films were sputtered for $2 \mathrm{~h}$. Sputtering rate determination was carried out using a quartz crystal microbalance.

SEM/EDX analysis was carried out using a JEOL JSMIT100 operated at $20 \mathrm{kV}$, with probing current at $70 \%$ for 2 min per measurement, leading to a total duration of $80 \mathrm{~min}$. Elemental compositions were quantified at $3000 \times$ magnification, corresponding to an area of $31 \times 23 \mu \mathrm{m}^{2}$. Low vacuum mode ( $35 \mathrm{~Pa}$ pressure) was used to facilitate quantitative elemental analysis without a conductive coating.

The sample stage of the XY-scanner was made out of two stacked Thorlabs DDSM100 linear translation stages. An Ekspla NT230 OPO Laser was used as excitation source. Two polarizers and a $500 \mu \mathrm{m}$ diaphragm were placed in the laser beam to reduce intensity of the laser light and to avoid fluctuations of the beam position. The intensity was reduced to prevent saturation of the luminescent centers in the sample. Four \% of the incident laser light was split off the main beam by a UV fused silica plate. This split-off light was directed toward a Thorlabs S120VC sensor connected to a Thorlabs PM100D calibrated powermeter for online correction of the laser power. The main laser beam was focused onto the film using a $f=100.3 \mathrm{~mm}$ Thorlabs LA4380 lens. Emission passed through a $355 \mathrm{~nm}$ long-pass filter (Semrock, BLP01-355R25 ), followed by a $f=100.3 \mathrm{~mm}$ Thorlabs LA4380 lens. This lens focused the emission into an Ocean Optics QP600-2-SRBX fiber. Emission was integrated during $100 \mathrm{~ms}$ and averaged 5 times using an Ocean Optics QE65000 CCD Spectrometer (100 $\mu \mathrm{m}$ slit, 300 grooves $/ \mathrm{mm}$ grating, leading to an uncertainty in emission wavelength of $\pm 1.69 \mathrm{~nm}$ ) and corrected for the quantum efficiency and nonlinearity of the detector. Prior to any emission measurement, the background was measured using identical settings and subtracted from the final measurement, leading to a measurement duration of $1 \mathrm{~s}$. $32 \times 32(\Delta x=1.56 \mathrm{~mm}, \Delta y=1.56 \mathrm{~mm})$ emission measurements were conducted across the entire substrate, leading to a total measurement duration of $17 \mathrm{~min}$.

For decay studies, the emission passed through an Acton Spect-Pro2300 monochromator coupled to a Hamamatsu R7600U-03 PMT, with the PMT linked to a CAEN DT5730 Digitizer. The digitizer was constantly recording, but started storing information upon reception of a trigger signal from the laser. When a trigger was received by the digitizer, the output of the PMT was stored ranging from $\sim 0.6 \mu$ s before the start of a laser pulse and to $\sim 54.6 \mu \mathrm{s}$ after a laser pulse, with a resolution of $\Delta t=2 \mathrm{~ns}$. Decays were recorded using 1000 laser pulses, resulting in an error for the calculated mean decay time below $1 \%$ (typically below $\pm 7 \mathrm{~ns}$ ). With the $100 \mathrm{~Hz}$ OPO laser, this yields $10 \mathrm{~s}$ per measurement. Here, measurements were conducted across the substrate with an $8 \times 8$ grid, yielding a total duration of $11 \mathrm{~min}$. All devices pertaining to the $\mathrm{XY}$-scanner were controlled using in-house developed Python software.

XRD measurements were performed using a PANalytical X'pert Pro MPD diffractometer with a $\mathrm{Cu} \mathrm{K} \alpha$ anode $(\lambda=$ $0.1540598 \mathrm{~nm})$ operating at $45 \mathrm{kV}$ and $40 \mathrm{~mA}$ in a BraggBrentano geometry measured from $2 \theta=20^{\circ}$ to $70^{\circ}, \Delta 2 \theta=$ $0.0083731^{\circ}$ in $1 \mathrm{~h}$. The area illuminated by the X-ray beam was around $1 \times 5 \mathrm{~mm}^{2}$ in size. The film was placed in a hygroscopic sample holder. The measurement locations on the film were set using a manually operated homemade $x y$ micromanipulator stage.

\section{ASSOCIATED CONTENT}

\section{Supporting Information}

The Supporting Information is available free of charge on the ACS Publications website at DOI: 10.1021/acscombsci. 8 b00068.

Comparative excitation and emission measurements of a common $\mathrm{Y}_{2} \mathrm{Al}_{5} \mathrm{O}_{12}: 0.01 \mathrm{Ce}^{3+} \mathrm{LED}$ phosphor, photographs (UV/visible), position-dependent decay spectra, and estimated quantum yields of the pure $\mathrm{NaI}: \mathrm{Eu}^{2+}$ and $\mathrm{NaBr}: \mathrm{Eu}^{2+}$ films (PDF)

\section{AUTHOR INFORMATION}

\section{Corresponding Author}

*E-mail: e.p.j.merkx@tudelft.nl.

ORCID

Evert P. J. Merkx: 0000-0003-4868-8409

\section{Author Contributions}

E.M. and E.K. conceived and designed the experiments, E.M. performed the experiments, and E.M. and E.K. cowrote the manuscript.

\section{Notes}

The authors declare no competing financial interest.

\section{ACKNOWLEDGMENTS}

This work was supported by The Netherlands Organization for Scientific Research (NWO/OCW), as part of the Frontiers of 
Nanoscience program (NF16NFS01) and as part of the LumiCon project (15024).

\section{REFERENCES}

(1) Park, W. B.; Singh, S. P.; Yoon, C.; Sohn, K.-S. Eu ${ }^{2+}$ luminescence from 5 different crystallographic sites in a novel red phosphor, $\mathrm{Ca}_{15} \mathrm{Si}_{20} \mathrm{O}_{10} \mathrm{~N}_{30}: \mathrm{Eu}^{2+}$. J. Mater. Chem. 2012, 22, 14068.

(2) Danielson, E.; Golden, J. H.; McFarland, E. W.; Reaves, C. M.; Wu, X. D.; Weinberg, W. H. A combinatorial approach to the discovery and optimization of luminescent materials. Nature 1997, 389, 944-948.

(3) Danielson, E.; Devenney, M.; Giaquinta, D. M.; Golden, J. H.; Haushalter, R. C.; McFarland, E. W.; Poojary, D. M.; Reaves, C. M.; Weinberg, W. H.; Wu, X. D.; Damodara, M.; Poojary, C. M. R.; Weinberg, W. H.; Wu, X. D. A Rare-Earth Phosphor Containing OneDimensional Chains Identified Through Combinatorial Methods. Science 1998, 279, 837-839.

(4) Sun, X.-D.; Gao, C.; Wang, J.; Xiang, X.-D. Identification and optimization of advanced phosphors using combinatorial libraries. Appl. Phys. Lett. 1997, 70, 3353-3355.

(5) Wei, Q.; Wan, J.; Liu, G.; Zhou, Z.; Yang, H.; Wang, J.; Liu, Q. Combinatorial Optimization of La, Ce-co-Doped Pyrosilicate Phosphors as Potential Scintillator Materials. ACS Comb. Sci. 2015, 17, 217-223.

(6) Takahashi, R.; Kubota, H.; Murakami, M.; Yamamoto, Y.; Matsumoto, Y.; Koinuma, H. Design of Combinatorial Shadow Masks for Complete Ternary-Phase Diagramming of Solid State Materials. J. Comb. Chem. 2004, 6, 50-53.

(7) Lee, B.; Lee, S.; Jeong, H. G.; Sohn, K.-S. Solid-State Combinatorial Screening of $(\mathrm{Sr}, \mathrm{Ca}, \mathrm{Ba}, \mathrm{Mg})_{2} \mathrm{Si}_{5} \mathrm{~N}_{8}: \mathrm{Eu}^{2+}$ Phosphors. ACS Comb. Sci. 2011, 13, 154-158.

(8) Chen, L.; Fu, Y.; Zhang, G.; Bao, J.; Gao, C. Optimization of $\mathrm{Pr}^{3+}, \mathrm{Tb}^{3+}$, and $\mathrm{Sm}^{3+}$ Co-Doped $\left(\mathrm{Y}_{0.65} \mathrm{Gd}_{0.35}\right) \mathrm{BO}_{3}: \mathrm{Eu}_{0.05}{ }^{3+} \mathrm{VUV}$ Phosphors through Combinatorial Approach. J. Comb. Chem. 2008, 10, 401-404.

(9) Chan, T.-S.; Liu, Y.-M.; Liu, R.-S. Combinatorial Search for Green and Blue Phosphors of High Thermal Stabilities under UV Excitation Based on the $\mathrm{K}\left(\mathrm{Sr}_{1-\mathrm{x}-\mathrm{y}}\right) \mathrm{PO} 4: \mathrm{Tb}_{\mathrm{x}}{ }^{3+} \mathrm{Eu}_{\mathrm{y}}{ }^{2+}$ System. J. Comb. Chem. 2008, 10, 847-850.

(10) Frost, S.; Guérin, S.; Hayden, B. E.; Soulié, J.-P.; Vian, C. HighThroughput Synthesis and Characterization of Eu Doped $\mathrm{Ba}_{x} \mathrm{Sr}_{2-x} \mathrm{SiO}_{4}$ Thin Film Phosphors. ACS Comb. Sci. 2018, 20, 451460.

(11) Wu, J. L.; Danielson, E.; DenBaars, S. P.; Devenney, M.; McFarland, E. W.; Srdanov, V. I.; Weinberg, H. Proc. SPIE 2001, 7081.

(12) Yano, K.; Takeshita, S.; Iso, Y.; Isobe, T. Combinatorial optimization of the atomic compositions for green-emitting $\mathrm{YBO}_{3}: \mathrm{Ce}^{3+}, \mathrm{Tb}^{3+}$ and red-emitting $\mathrm{YBO}_{3}: \mathrm{Ce}^{3+}, \mathrm{Tb}^{3+}, \mathrm{Eu}^{3+}$ phosphors using a microplate reader. RSC Adv. 2017, 7, 17586-17592.

(13) Fowlkes, J. D.; Fitz-Gerald, J. M.; Rack, P. D. Ultraviolet emitting $\left(\mathrm{Y}_{1-x} \mathrm{Gd}_{x}\right)_{2} \mathrm{O}_{3}-\delta$ thin films deposited by radio frequency magnetron sputtering: Combinatorial modeling, synthesis, and rapid characterization. Thin Solid Films 2006, 510, 68-76.

(14) Rubio O., J. Doubly-valent rare-earth ions in halide crystals. J. Phys. Chem. Solids 1991, 52, 101-174.

(15) Siegel, G. Ultraviolet spectra of silicate glasses: A review of some experimental evidence. J. Non-Cryst. Solids 1974, 13, 372-398.

(16) de Jong, M.; Kesteloo, W.; van der Kolk, E. Deposition of luminescent $\mathrm{NaCl}: \mathrm{Tm}^{2+}$ thin films with a Tm concentration gradient using RF magnetron sputtering. Opt. Mater. 2015, 46, 149-153.

(17) Ohring, M. Materials Science of Thin Films; Academic Press, 2001; pp 95-201.

(18) Nakazawa, E. In Phosphor Handbook, 2nd ed.; Yen, W. M., Shionoya, S., Yamamoto, H., Eds.; CRC Press, 2006; Chapter 2.8, pp 99-110.

(19) Muñoz, G. H.; de la Cruz, C. L.; Muñoz, A. F.; Rubio, J. O. High-temperature luminescence properties of $\mathrm{Eu}^{2+}$-activated alkali halide phosphor materials. J. Mater. Sci. Lett. 1988, 7, 1310-1312.
(20) Dorenbos, P. Relation between $\mathrm{Eu}^{2+}$ and $\mathrm{Ce}^{3+} \mathrm{f} \leftrightarrow \mathrm{d}$-transition energies in inorganic compounds. J. Phys.: Condens. Matter 2003, 15, 4797-4807. 